\title{
Modélisation probabiliste des efforts de houle sur une structure offshore en présence de bio salissures
}

\author{
Boukinda M. ${ }^{(a)}$, Schoefs F ${ }^{\text {(a) }}$, Belorgey M. ${ }^{(b)}$ \\ (a)GeM-UMR-CNRS 6183, Université de Nantes, Faculté des Sciences et Techniques, \\ B.P. 92208, 44322 Nantes cedex 3, France, E-mail :schoefs@physique.univ-nantes.fr. \\ (b)UnM2C-UMR-CNRS 6143, Groupe Mécanique des Fluides, 24, Rue des Tilleuls ; F-14000 Caen
}

\section{Résumé}

L'article se concentre sur l'effet des bio-salissures marines sur les efforts hydrodynamiques sur des structures offshore de type Jacket. Dans un contexte de fiabilité des structures, une modélisation probabiliste des coefficients de traînée du modèle de Morison est présentée. Elle est basée sur le conditionnement aux variables de houle, via les paramètres Kc et Re. Une illustration est basée surune colonisation par des moules et une étude en situation de tempête en mer du Nord.

\section{Abstract}

Structural response to extreme events or fatigue loading and structural integrity are major criteria to be quantified in a rational process of reassessment for offshore structures. It is now well established that the probabilistic mechanics approach gives an efficient means for measuring the relative changing in safety level compared to a predefined requirement. This paper proposes a probabilistic modelling of marine growth effects based on both Response Surface Methodology and the panel of available tests in laboratory. This method is here suggested to condition hydrodynamics coefficient to particle kinematics field.

Mots clés: structures offshore, modélisation probabiliste, fiabilité, coefficient hydrodynamiques, bio-salissures.

Keywords: offshore structures, probabilistic modelling, reliability, hydrodynamic coefficients, marine growth.

\section{Introduction}

Depuis trois décennies, le phénomène de colonisation des structures par des organismes marins, végétaux ou animaux, suscite un intérêt particulier en vue d'analyser leur impact sur les chargements hydrodynamiques. Ce phénomène appelé bio-salissures, longtemps sous estimé, provoque aussi des dégradations mécaniques et chimiques qui modifient le comportement de la structure ${ }^{1}$. De nombreux travaux ont été effectués afin de caractériser et de mesurer le phénomène de colonisation et ses effets hydrodynamiques. Les pratiques actuelles peuvent être regroupées en deux familles qui permettent d'aborder la question de modélisation : la modélisation hydrodynamique expérimentale et la modélisation physique des bio-salissures à partir des mesures in-situ².

Concernant la première approche deux auteurs peuvent être considérés comme des référentiels. Les travaux de Sarpkaya ${ }^{3,4}$, sur des cylindres fixes rugueux et lisses en écoulement sinusoïdal, ont démontré que la surface de rugosité a une influence sur les coefficients de forces de l'équation de Morison (coefficient de traînée et d'inertie) et 
l'écoulement. Théophanatos ${ }^{5}$ montre que la nature des bio-salissures peut être déterminante pour le choix des coefficients de forces en calcul d'efforts.

Dans la seconde approche, les données issues des campagnes de mesures sont exploitées en phase de la conception pour prédire l'expansion et le type de bio salissures et durant l'exploitation pour optimiser les procédures de nettoyage en estimant la quantité de biosalissures. Les informations rapportées permettent également de modéliser les effets majeurs générés par les bio-salissures.

La modélisation probabiliste proposée est utilisée afin de prendre en considération le caractère aléatoire des variables de base (hauteur et période de houle) mais aussi les incertitudes de modèle liées au coefficient de traînée (essais expérimentaux) et aux biosalissures. Quelques notions sur les impacts majeurs des bio salissures au niveau du modèle de Morison et des structures marines sont rappelées à cette fin.

\section{$\underline{2}$ Enjeux liés à la présence de bio salissures}

De multiples études, Faber $^{6}$, Schoefs ${ }^{7} . .$. , montrent que les bio-salissures génèrent des effets importants sur la durée d'exploitation d'une structure. Ces derniers, difficilement quantifiables compte tenu du caractère aléatoire du processus de colonisation et de la diversité des espèces présentes, engendrent des incertitudes sur les chargements hydrodynamiques. Le modèle de Morison, généralement proposé en calcul d'effort, est de ce fait entouré de nombreux aléas liés aux coefficients hydrodynamiques (traînée et inertie) et à la prise en compte des bio-salissures. Les incertitudes relatives au modèle de Morison sont abordées ainsi qu'un bref aperçu des effets des bio-salissures afin de cerner les différentes sources d'incertitudes et de quantifier leur niveau en vue d'une modélisation.

\subsection{Modèle de Morison}

Dans les équations de Morison, les chargements hydrodynamiques agissant sur les éléments structuraux résultent d'une somme des forces de traînée et d'inertie ${ }^{8}$ :

$$
\mathrm{F}(\mathrm{t})=\mathrm{F}_{\mathrm{D}}+\mathrm{F}_{\mathrm{I}}=\frac{1}{2} \rho \mathrm{D} \theta_{\mathrm{mg}}\left[\mathrm{C}_{\mathrm{D}} \mathrm{u}(\mathrm{t})|\mathrm{u}(\mathrm{t})|+\frac{1}{2} \mathrm{C}_{\mathrm{M}} \pi \mathrm{Du}(\mathrm{t})\right]
$$

$F_{D}$ et $F_{I}$ : forces de traînée et d'inertie, $C_{D}$ et $C_{M}$ : coefficients de traînée et d'inertie

$\theta_{\mathrm{mg}}$ : facteur prenant en compte les bio salissures.

$\theta_{\mathrm{mg}}=1+2 \mathrm{t} / \mathrm{D}: \mathrm{t}$, épaisseur de bio-salissures ; D, diamètre d'origine du cylindre.

Les coefficients hydrodynamiques permettent de caractériser l'interaction fluide-structure et le facteur $\theta_{\text {mg }}$ prend en compte l'impact des bio-salissures. On montre que l'incertitude sur le diamètre des éléments structuraux colonisés engendre une incertitude sur le coefficient de traînée en raison des modifications hydrauliques générées par l'augmentation de diamètre associée. Les aléas liés au champ cinématique des particules, issues notamment de la prédiction de la houle extrême et de ses caractéristiques (hauteurs, périodes, et profil de surface), influencent également la variation des coefficients hydrodynamiques. Une sélection appropriée des coefficients introduits dans l'équation de Morison doit tenir compte des incertitudes afférentes.

\subsection{Impacts des bio salissures marines sur une structure de type Jacket}

Les bio-salissures influencent les chargements hydrodynamiques de manières différentes. Plusieurs auteurs (Théophanatos ${ }^{5}$, Picken ${ }^{1} . .$. ) ayant fournies des études complètes sur ces effets, seuls les principaux sont rappelés dans ce papier. 


\subsubsection{Accroissement diamétral des éléments structurels}

La diversité des communautés de bio-salissures et la compétitivité à laquelle elles se livrent au cours du processus de colonisation entraînent une stratification de couches sur les membres structuraux. L’accroissement diamétral généré (jusqu'à 30\%) provoque une augmentation substantielle du chargement hydrodynamique mais aussi crée un effet de masque pour les inspections de la structure.

\subsubsection{Modification des efforts hydrodynamiques}

Théophanatos ${ }^{5}$ et Sarpkaya ${ }^{3}$ montrent que les bio salissures induisent des effets d'écran se traduisant par une augmentation des efforts hydrodynamiques et du coefficient de traînée sur les surfaces exposées. Sur la figure 1, le coefficient de traînée est en moyenne deux fois plus important pour des cylindres rugueux (partie asvmptotique des courbes) et croît avec la rugosité relative $\mathrm{k} / \mathrm{D}$, $\mathrm{k}$ étant la rugosité et

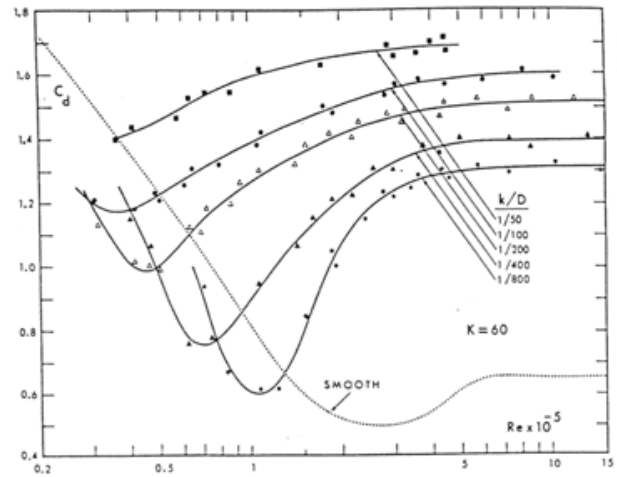

Figure 1 : Evolution du coefficient de traînée en fonction du nombre de Reynolds et de la rugosité relative.

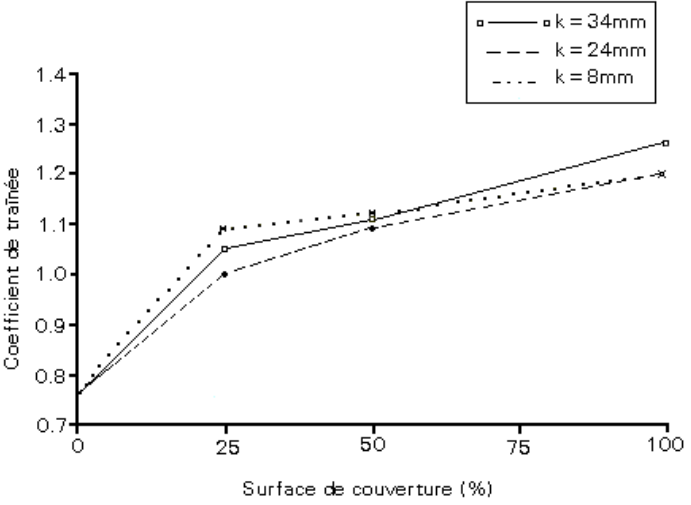

Figure 2 : Evolution du coefficient de traînée en fonction du pourcentage de surface couverte, pour différentes rugosités.

Le pourcentage de couverture des bio-salissures influe également sur le niveau des coefficients hydrodynamiques. La figure 2 montre un accroissement du coefficient de traînée de 0.78 à 1.05 pour $25 \%$ de couverture de moules à 1.2 pour $100 \%$ de couverture.

\subsubsection{Modification de l'écoulement}

Les essais expérimentaux de Achenbach $^{5}$ révèlent qu'à rugosité relative croissante, la limite critique se déplace vers de faibles valeurs de Reynolds. Ce constat est aussi établi par Buresti ${ }^{9}$ qui démonte que pour des valeurs de $\mathrm{Re}_{\mathrm{k}}$ (nombre de Reynolds rugueux) supérieures à 200, il était possible d'atteindre un régime super-critique. Les bio-salissures marines modifient donc la fréquence tourbillonnaire autour des piles principales de la structure altérant le régime d'écoulement et les coefficients hydrodynamiques.

\section{Modélisation du coefficient de traînée}

Une approche pragmatique est proposée afin d'évaluer des coefficients mieux adaptés au calcul d'efforts. L'étude se concentre sur des éléments cylindriques horizontaux et verticaux d'une structure jacket en condition de tempête située en Mer du Nord. Etant donné la prolifération de bio-salissures entre 0-50 mètres de profondeur et l'importance de l'intensité des composantes (vitesse et accélération) du champ cinématique particulaire dans la zone de la vague, l'étude n’est réalisée que sur cette tranche d'eau. 
Un effet de projection des vitesses sur les éléments horizontaux, verticaux ou inclinés est pris en compte dans le programme de

calcul Malost ${ }^{7}$, basé sur une modélisation par surface de réponse du champ cinématique et des efforts de houle.

\subsection{Champ stochastique des paramètres hydrauliques (Re et $\mathrm{Kc})$}

Les statistiques moyennes des trajectoires des paramètres hydrauliques, pour des cylindres verticaux de 1 mètre de diamètre, sont visualisées en figures 3 et 4 . Elles ont été obtenues à partir des bases de donnée des paramètres de houle (hauteur et période) caractérisant l'état de mer de tempête ${ }^{10}$. Re décroît de 5.6 à $1.7^{*} 10^{6}$ dans la direction de propagation de la houle et de 5.6 à $2.7 * 10^{6}$ environ avec la profondeur. Kc varie de 83 à 26 environ. Ces évolutions se retrouvent sur les simulations de Kc et Re effectuées pour des cylindres horizontaux ${ }^{11}$. Le caractère périodique de la houle définit un axe de symétrie à 80 mètres qui permet de restreindre le domaine d'étude. Ces moyennes montrent une forte variabilité mais ne permettent pas de caractériser précisément les domaines de variations. Une étude exhaustive a été réalisée sur les autres statistiques. En particulier, afin de caractériser plus précisément les densités, les coefficients d'asymétrie (skewness) présentées en figure 5 et 6 montrent une forte variabilité en fonction de la profondeur et de la direction de propagation de la houle.

Les coefficients négatifs et positifs indiquent des distributions asymétriques. Les coefficients proches de 0 mentionnent des distributions qui peuvent être associées a des lois normales. On constate qu'en dehors de l'allure des iso-valeurs, les valeurs des coefficients sont très différentes entre Re et Kc. Ceci provient notamment de leur expression en fonction de la vitesse. Schoefs et Labeyrie ${ }^{12}$ démontrent que la hauteur et la période de la houle influencent respectivement le caractère asymétrique et symétrique des distributions avec des contributions diverses en fonction de la profondeur. Afin d'effectuer une modélisation pour un éléments structurel, vertical ou horizontal, il est nécessaire ensuite de regrouper ces données par tranches d'eau.

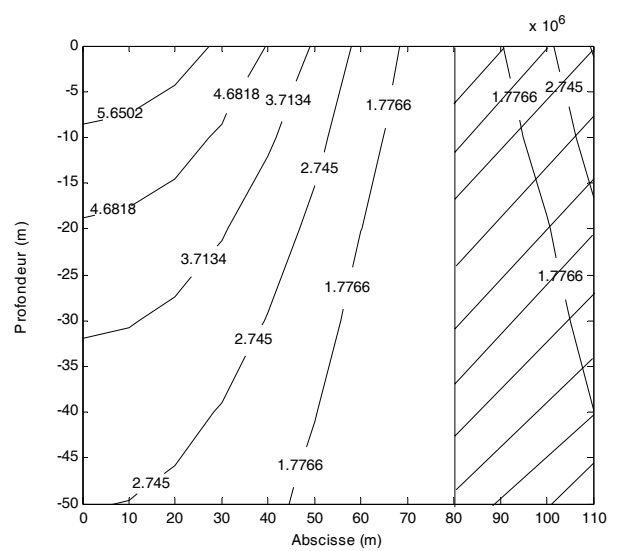

Figure 3 : Carte d'iso-valeurs des nombres Re (cylindre vertical)

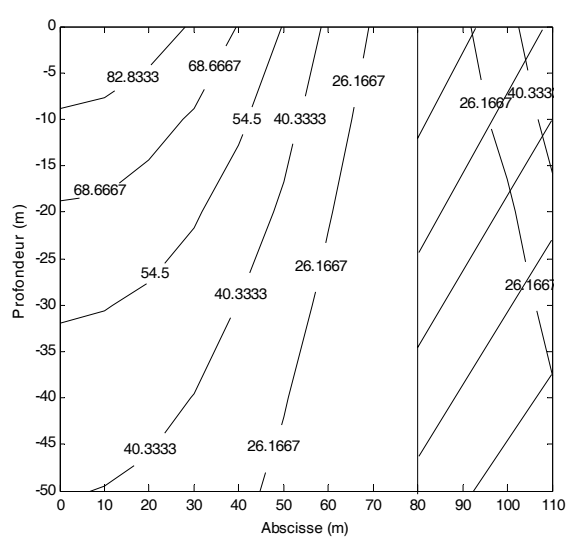

Figure 4 : Carte d'iso valeurs des nombres KC (cylindre vertical) 


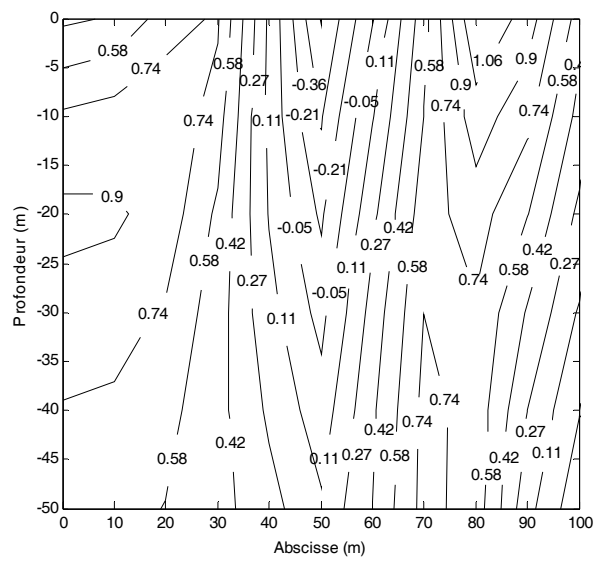

Figure 5 : Distribution du skewness de Re pour un cylindre vertical

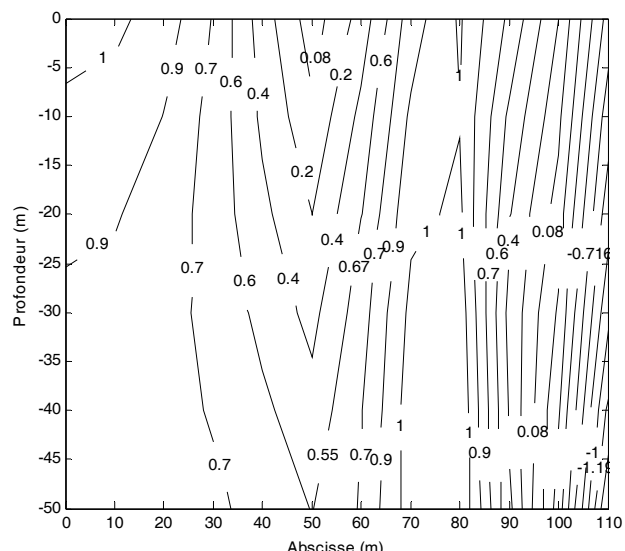

Figure 6 : Distribution du skewness de Kc pour un cylindre vertical

\section{2.Évaluation du coefficient de traînée}

Pour une modélisation plus réaliste tenant compte les variabilités en profondeur, on travaille sur des tranches d'eau de $10 \mathrm{~m}$ d'épaisseur. Les données de Wolfram ${ }^{13}$ (en mer du Nord) sur une colonisation de moules de 50mm d'épaisseur moyenne et de 13,4 mm de coefficient de variation, située entre la surface et 10 mètres de profondeur conviennent pour caractériser l'accroissement diamétral. Le calcul des paramètres hydrauliques associés à la présence de bio salissures permet d'évaluer le coefficient de traînée à partir des résultats graphiques fournis par Théophanatos d'une part et le règlement API d'autre part. Ils sont données par les équations :

$$
\begin{aligned}
& \operatorname{Re}_{\mathrm{mg}}=\frac{\mathrm{U} \times \mathrm{De}}{v}=\theta_{\mathrm{mg}} \times \operatorname{Re} \\
& \mathrm{Kc}_{\mathrm{mg}}=\frac{\mathrm{U} \times \mathrm{T}}{\mathrm{De}}=\frac{\mathrm{Kc}}{\theta_{\mathrm{mg}}}
\end{aligned}
$$

avec $\mathrm{De}=\theta_{\mathrm{mg}} \times \mathrm{D}$, De diamètre effectif de l'élément; $\mathrm{U}$, projection de la vitesse particulaire perpendiculairement à l'axe de l'élément

L'étude basée sur les expériences de Théophanatos consiste à évaluer les coefficients hydrodynamiques suivant la nature des bio-salissures en s'attachant notamment à montrer et évaluer le niveau de dispersion des résultats obtenus selon les auteurs et les variations des

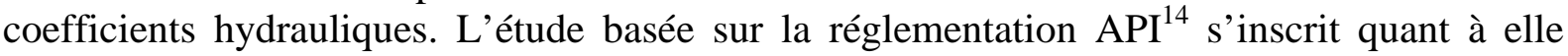
dans un cadre plus déterministe. On utilise la valeur de la moyenne des coefficients hydrauliques pour évaluer des coefficients moyens à partir de courbes de référence.

Considérons tout d'abord les coefficients Re et Kc des équations 2 et 3 comme des valeurs moyenne sur le champ stochastique. On réalise une première étude de sensibilité en considérant les valeurs moyennes et extrêmes de $\theta_{\mathrm{mg}}$ à partir des valeurs d'épaisseur moyenne et de l'écart type. Si on considère que l'épaisseur suit une loi normale ces valeurs sont obtenues pour $(\mu-3 \sigma)$ et $(\mu+3 \sigma)$ où $\mu$ et $\sigma$ désignent, comme dans la suite de l'article la moyenne et l'écart type. Sur la figure 7 , on peut constater que les statistiques du coefficient de traînée sont plus importantes et moins dispersées lorsqu'elles sont déterminées à partir des données fournies par Théophanatos. Le facteur $\theta_{\mathrm{mg}}$ étant soumis à de fortes variabilités (mesures incertaines de l'épaisseur de bio salissures), la création d'une base de données tenant 
compte de la nature des bio-salissures reste primordiale. En outre, il apparaît que le coefficient de traînée augmente avec le diamètre. Par la suite seul un diamètre de 1 mètre est considéré et cet effet n'est pas pris en compte afin de ne conserver que l'effet des aléas sur les variables de houle, prépondérantes.

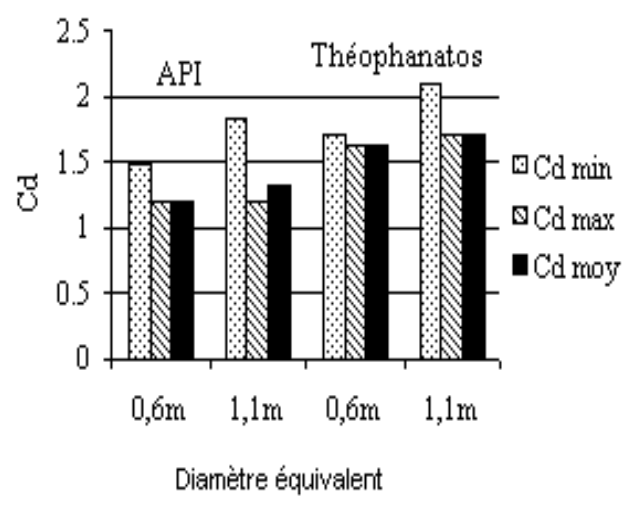

Figure 7 : Variation de $C_{D}$ en fonction du diamètre équivalent moyen.

\section{Modélisation probabiliste du coefficient de traînée}

Les analyses en fiabilité des structures offshore, qui nécessitent d'évaluer le chargement hydrodynamique sur une structure, utilisent en général un modèle aléatoire prédéfini (loi normale avec un coefficient de variation de 30\% pour le coefficient de traînée). Les variabilités inhérentes aux paramètres majeurs (hauteur et période de houle) qui influencent la réponse hydrodynamique ne sont pas souvent prises en compte au niveau du coefficient de traînée. Pourtant les recherches de Schoefs ${ }^{10}$ montrent la grande variabilité des statistiques des efforts sur les hypothèses statistiques du coefficient de traînée. En particulier il a été montré que le coefficient de variation des efforts pouvait varier en mer du Nord de 22 à $36 \%$ pour un coefficient de variation sur Cd variant de 15 à $30 \%$. Le modèle probabiliste proposé en figure 8 est utilisé afin de prendre en considération le caractère aléatoire des variables de base (hauteur et période de houle) mais aussi les incertitudes liées au coefficient de traînée (essais expérimentaux) et aux bio salissures (facteur $\theta_{\mathrm{mg}}$ ). On conditionne alors Cd au paramètre hydraulique prépondérant, ici Kc. Les illustrations seront données pour une barre verticale située dans la tranche d'eau $0-10 \mathrm{~m}$.

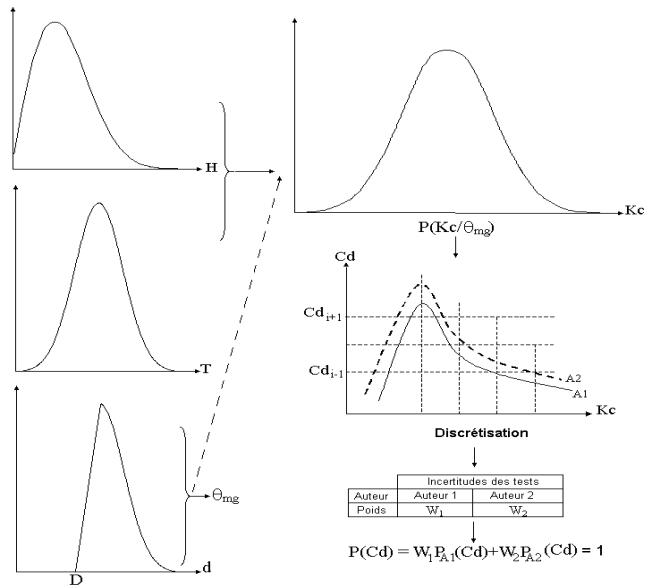

Figure 8 : Schéma de la modélisation probabiliste de Cd.

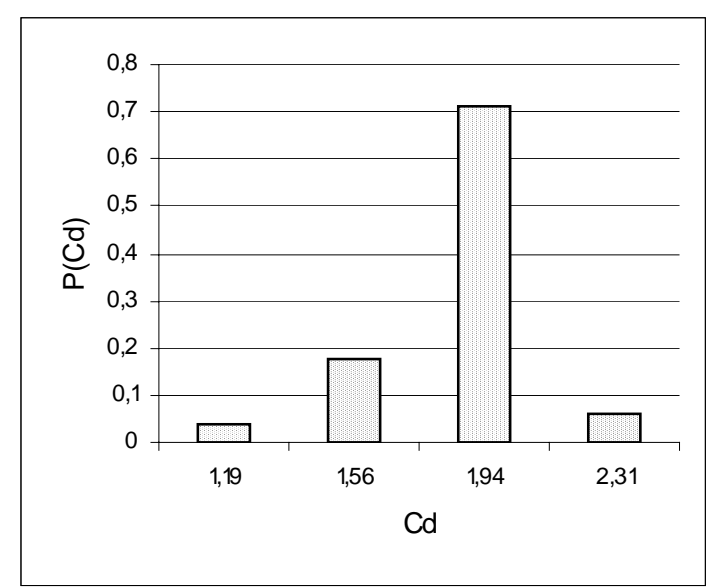

Figure 9 : Modélisation probabiliste de Cd conditionnellement à $\mathrm{Kc}$ 
Les variables aléatoire de houle (hauteur et période) et la variable $\theta_{\mathrm{mg}}$ (ici prise à sa valeur moyenne) permettent de caractériser le champ stochastique et d'obtenir les probabilités des paramètres hydrauliques ${ }^{2}$. Comme indiqué dans le paragraphe 3 , selon que l'on se base sur une approche réglementaire (API) ou empirique (Théophanatos), plusieurs modélisations peuvent être envisagées :

- Une première étude, basée règlement API, consiste à déterminer la moyenne des coefficients hydrauliques et d'en déduire un $\mathrm{Cd}$ moyen à partir du règlement pour enfin réaliser une modélisation probabiliste a priori sur la base d'une loi normale et d'un coefficient de variation de $30 \%$ préconisé dans la littérature ${ }^{16}$.

- Une deuxième approche, toujours effectuée par rapport au règlement API consiste à utiliser une loi conforme aux dépouillements statistiques c'est à dire une loi de Gumbel. On conserve alors un coefficient de variation de $30 \%$.

- Un dernier choix, développé dans cet article, suit la procédure de la figure 8 sur la base des expériences de Théophanatos. Cette figure présente le cas général mais les illustrations qui suivent sont basées sur une valeur de $\theta_{\mathrm{mg}}$ fixée à sa valeur moyenne. Compte tenu de la non linéarité des courbes, en première approche, l'étude discrète ${ }^{15}$ est basée sur une discrétisation des valeurs de Kc par tranches : 10-15 ; 16-21 ; 22-35.

On note alors, $\mathrm{P}\left(\mathrm{X}_{\mathrm{i}}\right)=\mathrm{P}\left(\mathrm{x}_{\mathrm{i}-1}<\mathrm{X}_{\mathrm{i}}<\mathrm{x}_{\mathrm{i}+1}\right)$

Selon le protocole suivi par chaque auteur et le mode de traitement des données choisi, un coefficient de pondération est ajusté. Il est maximal lorsqu'on estime avoir une très bonne corrélation entre les essais expérimentaux et les mesures de paramètres hydrauliques, ici le nombre de Keulegan-Carpenter. Cela permet d'enrichir la base de donnée en affectant une confiance aux séries de données.

$$
P\left(C d_{i}\right)=w_{1} \sum_{j=1}^{m} P_{A 1}\left(C d_{i} \mid K c_{j}\right) P\left(K c_{j}\right)+w_{2} \sum_{j=1}^{m} P_{A 2}\left(C d_{i} \mid K c_{j}\right) P\left(K c_{j}\right)
$$

On obtient bien une probabilité puisque :

$$
\mathrm{P}(\mathrm{Cd})=\sum_{\mathrm{i}=1}^{\mathrm{n}} \mathrm{P}\left(\mathrm{Cd}_{\mathrm{i}}\right)=1
$$

avec $\mathrm{w}_{1}+\mathrm{w}_{2}=1$. $\mathrm{n}$ provient des relations suivantes: $\mathrm{P}(\mathrm{Cd}<\mathrm{Cd} 1)=0, \mathrm{P}(\mathrm{Cd}>\mathrm{Cdn})=0$; ici $\mathrm{n}=4$ (voir figure 9). Ici $\mathrm{m}=3$ avec les tranches ${ }^{15} 10-15,16-21,22-35$.

La figure 9 indique que la distribution discrète est de type asymétrique ce qui est cohérent avec les coefficients d'asymétrie obtenus en section 3.1 pour les paramètres hydrauliques. La moyenne et l'écart-type de cette variable discrète sont obtenus à partir des équations (7) et (8) et valent respectivement $\mu=1.834$ et $\sigma=0.226$. Le coefficient de variation de $12 \%$ montre sur cet exemple que l'intervalle de 12 à 30\% généralement proposé en calcul de fiabilité ${ }^{\prime 6}$ est très conservatif.

$$
\begin{aligned}
& \mu(C d)=\sum_{i=1}^{4} P\left(C d_{i}\right) C d_{i} \\
& \sigma^{2}(C d)=\sum_{i=1}^{4} P\left(C d_{i}\right)\left(\mu(C d)-C d_{i}\right)^{2}
\end{aligned}
$$

Afin de caractériser la typologie de la distribution, il faut faire un choix parmi les fonctions de distributions disponibles. On effectue l'ajustement sur les fonctions de répartitions $\mathrm{F}_{\mathrm{Cd}}$, fonctions monotones, par la méthode des moindres carrés. On note, sur la 
figure 10, une bonne corrélation (erreur quadratique totale inférieure au millième) entre la fonction de répartition issue de la modélisation discrète et celle de le loi de Gumbel de type I (loi de valeur extrême de type I) de fonction de distribution donnée en (9). Cet ajustement est particulièrement adéquat sur la queue de distribution à droite, fondamentale pour le calcul en fiabilité. Les paramètres issus de l'optimisation sont $\alpha=1,79 ; \beta=0.16$ :

$$
f(x)=\frac{1}{\beta} \exp \left(\frac{x-\alpha}{\beta}\right) * \exp \left(-\exp \left(\frac{x-\alpha}{\beta}\right)\right)
$$

On exploite ces résultats selon les trois approches présentées plus haut :

- La probabilisation des données API, en prenant la valeur réglementaire obtenue pour la moyenne et un coefficient de variation de 30\% avec loi normale.

- La probabilisation des données API, avec la même base que celle ci-dessus mais cette fois la loi de Gumbel de type I.

- La probabilité discrète basée sur les données de Théophanatos (voir figure 8).

Les résultats, sous forme de densités de probabilité, sont présentés en figure 11. Le tableau 1 résume les paramètres statistiques du coefficient de traînée.

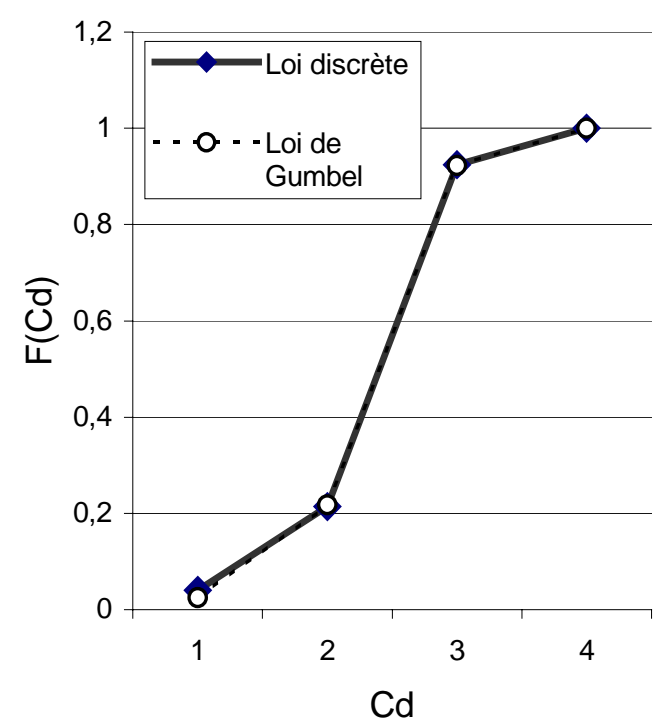

Figure 10 : Fonctions de répartition discrète de Cd et de la loi de Gumbel type I ajustée.

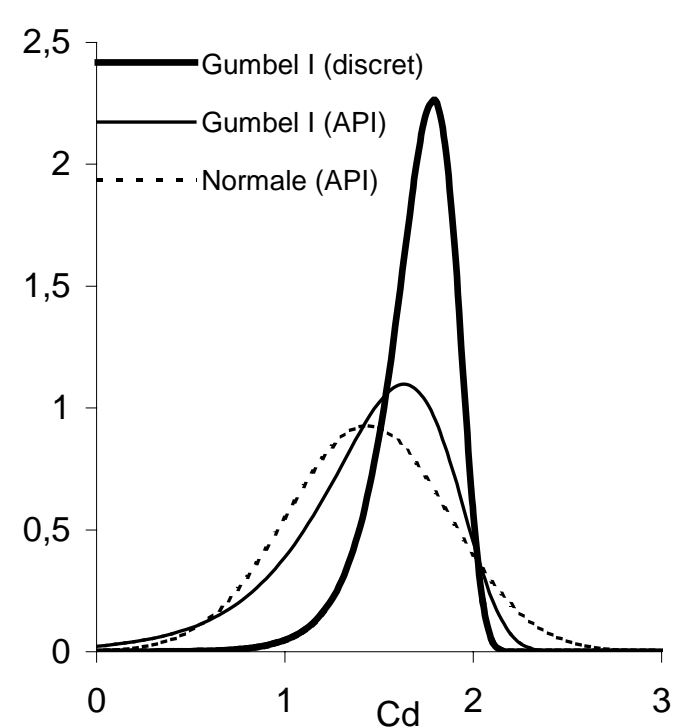

Figure 11 : Densité de probabilité obtenues par les trois approches.

Tableau 1 : Paramètres statistiques du coefficient de traîné

\begin{tabular}{|c|c|c|c|}
\hline & Moyenne & Ecart-type & Cov (\%) \\
\hline Gumbel discret & 1.69 & 0.21 & 12 \\
\hline Gumbel API & 1.44 & 0.43 & 30 \\
\hline
\end{tabular}

On note qu'en terme de maximum de probabilité, les modélisations Normale-API, Gumbel I-API et Gumbel I-discret conduisent respectivement à des coefficients plus importants. En raisonnant sur les queues de distribution, sur la partie droite, le constat diffère : l'approche Normale-API conduit à une majoration importante au niveau de la probabilité de queue de distribution mais est conservative. L'approche Gumbel I-API, même avec un CoV arbitraire, conduit à une représentation plus acceptable de la représentation basée sur une étude discrète. Ces densités de probabilités permettent de générer des calculs d’efforts. 


\section{Conclusion}

La modélisation probabiliste du chargement hydrodynamique sur une structure en présence de bio-salissures n'est pas aisée. Les incertitudes relatives aux coefficients hydrodynamiques de l'équation de Morison, aux paramètres hydrauliques et au champ cinématique particulaire demandent une caractérisation rigoureuse. On propose de baser la modélisation sur un conditionnement des coefficients hydrodynamiques aux paramètres de l'écoulement Kc et Re. Une illustration complète a été donnée pour le coefficient de traînée et montre l'intérêt d'une telle approche notamment pour caractériser la dissymétrie de sa densité de probabilité. On a montré qu'une approche réglementaire basée sur cette hypothèse était nettement plus satisfaisante pour un calcul en fiabilité.

\section{Références}

1.Picken G.B. (1985) "Review of marine fouling organisms in the North Sea on offshore structures”, Discussion Forum and Exhibition on Offshore Engineering with Elastomers, June 1985, Plastics and Rubber Inst., London , vol. 5, pp. 5.1 - 5.10.

2.Schoefs F. et Boukinda Morgan (2004) "Modelling of marine growth effect on offshore structures using kinematics field of water particle”, Proc. OMAE, pp 8

3.Sarpkaya T. (1977) "In-line and transverse forces on cylinders on cylinders in oscillatory flow at high Reynolds numbers”, Journal of Ship Research, Vol.21, N4, pp.200-216.

4.Sarpkaya T. (1990) "On the effect of roughness on cylinders" , Journal of Offshore Mechanics and Artic Engineering, Transactions of the American Society of Mechanical Engineers, Vol.112, pp.334-340.

5.Theophanatos A. (1988) "Marine growth and hydrodynamic loading of offshore structures", PhD thesis, University of Strathclyde - UK, Feb 1988.

6.Faber M.H., Hansen P.F., Jepsen F.D. and Moller H.H. (2001) "Reliability-based management of marine fouling”, Journal of Offshore Mechanics and Arctic Engineering, vol 123, Issue 2, pp. 76-83, Elsevier Science Ltd.

7.Schoefs F. (1996) "Response Surface of wave loading for reliability of marine structures”PhD Thesis, nov. 1996, University of Nantes.

8.Morison, J.R., O'Brien M.P., Johnson J.W. and Schaff. S.A. "The forces exerted by surfaces waves on piles", Petroleum trans.

9.Buresti B. (1978), "Vortex shedding from smooth and roughened cylinders in crossflow near a plane surface".

10.Labeyrie J. and F. Schoefs, (1995) “A formal geometrical modelling of wave actions for structural reliability”, Proc. of O.M.A.E, Copenhague, pp. 6.

11.Boukinda M. (2003), "Caractérisation mécanique des chargements hydrodynamiques dus aux bio salissures marines, ”Rapport interne, Université de Nantes.

12.Labeyrie, J. and F. Schoefs, (1996) "Matrix Response Surfaces For Describing Environmental Loads”, vol. II Safety and Reliability, Proc. of 15th O.M.A.E, Florence, pp. 119-126.

13.Wolfram J., Jusoh I. et Sell D. (1993) "Uncertainty in the estimation of the fluid loading due to the effects of marine growth", Safety and Reliability Symposium, Proc. of 12th int. conf. on Offshore Mechanics and Arctic Engineering, (O.M.A.E'93), Glasgow, vol. II, pp. 219-228 (ASME 1993).

14.API (1986) "Study on wave forces on a cylinder in the fields where wave and flow coexist”, Proc. Of International Offshore Mechanics and Arctic Symposium, Tokyo - Japan, pp. 130-135, ASME Pub. New York. 
15.Schoefs F. et Boukinda M. (2004) "Modelling of marine groxth effect on offshore structures loading using kinematics field of water particle", Proceeding of 14th I.S.O.P.E, 2328 mai 2004, Toulon, France, 8 pages, pp. 419-426.

16.Engineering Committee on Oceanic Resources (1991), "Reliability analysis for offshore structures”, pp.81. 